\title{
Nanofabrication of Robust Nanoelectrodes for Electrochemical Applications
}

\author{
K. Dawson ${ }^{\text {a }}$, J. Strutwolf ${ }^{\text {a }}$, G. Herzog ${ }^{\text {a }}$, D.W.M., Arrigan ${ }^{\mathrm{b}}$, A.J, Quinn ${ }^{\mathrm{a}}$, A. O’Riordan ${ }^{\text {a }}$ \\ a Tyndall National Institute. University College Cork, Lee Maltings, Cork, Ireland. \\ ${ }^{\mathrm{b}}$ Nanochemistry Research Institute, Department of Chemistry, Curtin University of \\ Technology, Perth, WA 6845, Australia.
}

\begin{abstract}
Individual gold nanowire devices for use as nanoelectrodes in electrochemical studies were fabricated using a low-cost robust fabrication approach. Nanowires were characterized by a combination of direct electrical current-voltage measurements and cyclic voltammetry using the model analyte ferrocene monocarboxylic acid. We observed low electrical resistances to contacted nanowires and steady-state sigmoidal electrochemical voltammograms that may be described by classical Butler-Volmer kinetics. The potential of nanowires for use in future biosensing applications was explored by demonstrating mediated bioelectrocatalytic oxidation of glucose.
\end{abstract}

The growing need for point of care (POC) technologies is driving the development of more sensitive and more advanced sensor devices, particularly nanosensors. POC technologies are becoming more pervasive and range from the simple home pregnancy test kit to more complex blood analyzers employed in emergency clinical environments. With the global nanosensor market expected to exceed $\$ 2.4$ billion by 2012 a number of blue chip companies are establishing market positions (1). To win a market share new emerging POC technologies must provide: convenience, rapid analysis (less than 5 minutes), ease of use, assay detection limits equivalent to or better than established clinical laboratory assays, at low cost (less than $\$ 10$ per assay) and have low maintenance requirements. To this end, significant opportunity therefore exists to develop new sensor devices that exploit low cost nano-manufacturing methods. To address this opportunity, nanosensors based on 1-D nanostructures such as silicon nanowires and carbon nanotubes are being explored as theses nanostructures offer the potential to achieve highlyintegrated sensors with direct electrical readout (2-4). In these sensor systems, the perturbation in the local electrostatic environment of a nanowire-based field effect transistor by a charged analyte causes an increase or decrease in conductance, which is employed as the sensor signal (2). Although elegant, these sensor technologies require complex heterogeneous integration approaches following synthesis and, depending on device architecture, may require the application of back gate voltages as high as $40 \mathrm{~V}$ to be applied (5).

By contrast, electrochemical sensors typically have much lower voltages $(-1.5-1.5$ V) requirements. Compared to traditional macroelectrodes, nanoscale electrodes have tremendous potential as electrochemical sensors exhibiting enhanced performance. As critical dimensions of the electrodes enter the micro and nano regime, radial analyte diffusion profiles dominate with a corresponding increase in mass transport rate, higher current densities giving rise to an increased $\mathrm{I}_{\mathrm{F}} / \mathrm{I}_{\mathrm{C}}$ ratio (due to smaller surface area), higher $\mathrm{S} / \mathrm{N}$ ratios and steady-state sigmoidal voltammograms. (6). However, widespread 
take-up of nanoscale electrodes has been limited by the lack of practical and effective methodologies for fabrication of robust nanoelectrodes.

Nanoelectrode ensembles have been fabricated using a variety of approaches including: template-directed synthesis of deposited metals into sub-micron pores of commercial membranes (7-8), self-assembly of metallic nanoparticles(9-10), nanowires (11-12) or carbon microspheres decorated with nanoparticles at macroelectrodes (13-14), and opening of vias, through a self-assembled monolayer (15-16) or block copolymer (17) insulation layer to an underlying planar electrode yielding individual electrode elements of $\sim 6 \mathrm{~nm}$ radius. A key limitation with nanoelectrodes ensembles is individual nanoelectrode elements are not diffusionally independent, i.e., diffusion layers surrounding each electrode overlap, leading to a decrease of the current density at each individual electrode. This results in depletion layers being generated near each individual nanoelectrode leading to a significant decrease of the effective electrode area. To address this limitation, nanoelectrode arrays have been fabricated whereby individual nanoelectrodes are sufficiently spaced to render them diffusionally independent from closest neighbors. Focused ion beam milling and lithography and etching have been employed to etch arrays of nanopores into insulating layers on macroelectrodes (18). Recently it has shown, however, that diffusion to nanoelectrode arrays is dominated by diffusion to those at the edge of the array for non-infinite arrays (19). However, it was found that the voltammetric sweep rate influenced the profile of the diffusion layers such that, at low and very high sweep rates, diffusion profiles to each nanoelectrode overlapped resulting in a planar diffusion profiles.

With respect to individual nanoelectrodes, a variety of different approaches to fabrication have been reported including: fabrication of nanoband electrodes by evaporating thin metal films onto glass substrates, encapsulation and exposure of film edges only (20), sequential etching of a microwire to a fine cone followed by insulation of all but the cone tip (21-24), fabrication of disc electrodes by deposition of carbon in a capillary tube (25), and pulling of glass capillaries containing sealed microwires (26-27). Although individual nanoelectrodes have been used to probe nanoscale electrochemical phenomena, major disadvantages associated with these electrodes include: the difficulty and length of time for fabrication, leaking of electrolyte though the seals, and the extremely small currents (10-100 pA) that may be achieved with them. Such small currents pose problems when the measured current is to be used as the signal in a sensor electroanalytical application.

\section{Experimental: Nanowire Fabrication and Integration}

Herein we explore individual gold nanowire (NW) devices for use as nanoelectrodes in electrochemical studies fabricated using a low cost robust fabrication approach capable of achieving comparatively high electrochemical currents (nA); see Figure 1a. Nanowires were fabricated using the nanoskiving technique recently pioneered by the Whitesides Group (28). Briefly, a thin film of gold was evaporated onto an epoxy substrate which was further encapsulated in epoxy to form a block. Following curing, sections were sliced from the block using ultramicrotomy to yield nanomembranes each containing one gold nanowire. The nanomembranes were then deposited onto an oxidized silicon chip substrate $\left(90 \mathrm{~nm}\right.$, thermal $\left.\mathrm{SiO}_{2}\right)$ bearing arrays of micron-scale binary alignment marks, which were used for subsequent overlay of top-contact 
electrodes using optical lithography. Following deposition, nanowires were liberated from the epoxy membrane by $\mathrm{O}_{2}$ plasma ashing. Nanowires with lengths of several hundred microns and widths and heights of $\sim 207 \pm 1 \mathrm{~nm}$ and $254 \pm 11 \mathrm{~nm}$, respectively, were routinely fabricated. Using the binary mark identifiers for alignment and registration, contact electrodes were overlaid using optical lithography, metal evaporation (Ti $10 \mathrm{~nm}, \mathrm{Au} 200 \mathrm{~nm}$ ) and liftoff. Finally, a layer of photoresist ( $1 \mu \mathrm{m})$ was spin coated on to the chip to serve as an insulating layer preventing unwanted electrochemical reactions occurring at the interconnection tracks. Optical lithography was then employed to open a trench over the nanowire; see Figure 1b. Finally, chips bearing electricallycontacted nanowires were assembled onto printed circuit boards, electrically contacted using wedge wire bonding ( $25 \mu \mathrm{m}$ aluminum wire) and the bonds protected by epoxy to complete device packaging. Control devices (electrodes overlaid onto substrates without a nanowire present) were fabricated in a similar manner.

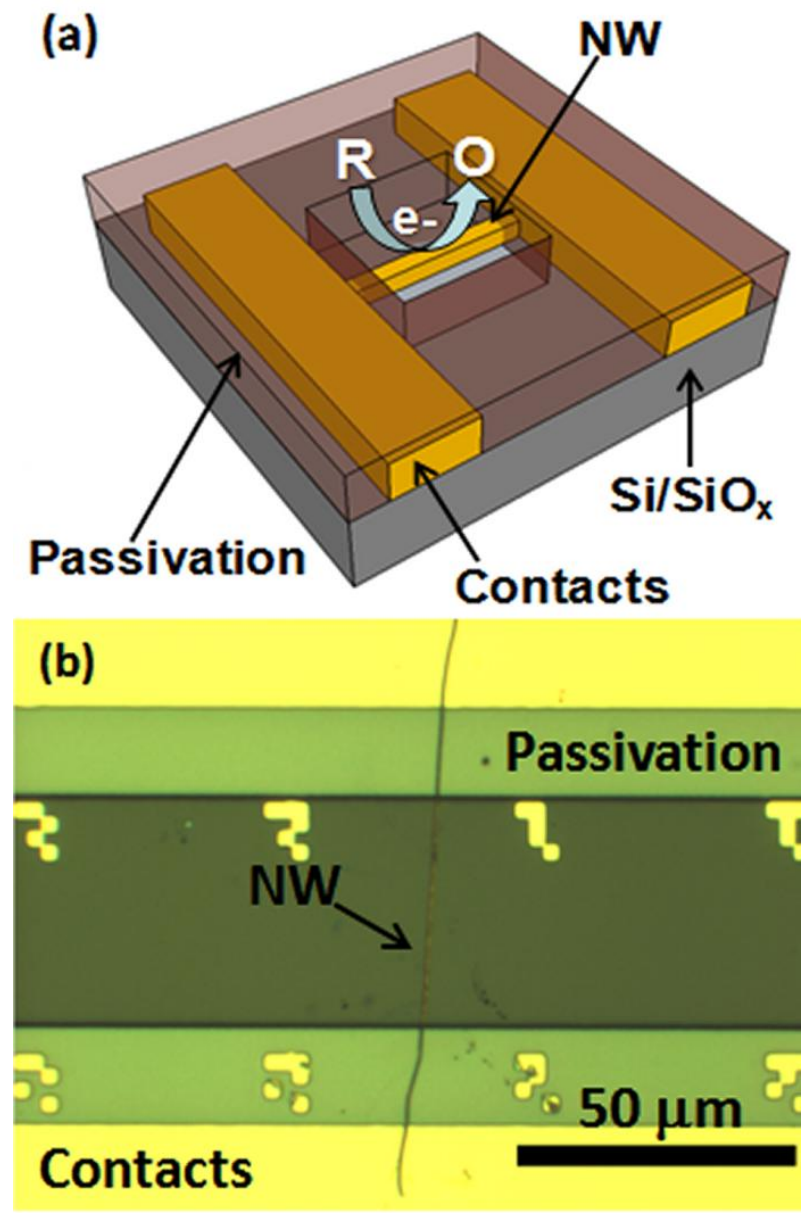

Figure 1. (a) Device schematic of a contacted gold nanowire. Contact electrodes were passivated using a photoresist insulating passivation layer. A trench was opened in the photoresist directly over the nanowire electrode to allow contact between the nanowire and the external environment. (b) Optical micrograph of a fully fabricated nanowire device. Scale bar, $50 \mu \mathrm{m}$. 


\section{Results:}

\section{Nanowire Characterization:}

Following fabrication, nanowires were characterized using a combination of currentvoltage (I-V) measurements and cyclic voltammetry, (CV). Two point electrical characterization of packaged devices demonstrated fully functional nanowires that exhibited very low resistance $\sim 80 \Omega(68-93 \Omega$ ) for $\sim 50 \mu \mathrm{m}$ long wires; see Figure $2 \mathrm{a}$. The measured track resistance was $16 \pm 2 \Omega$, ignoring contact resistances, a resistivity of $\sim 8 \times 10^{-8} \Omega \mathrm{m}$ was calculated for wires using a cross sectional area of $5.26 \times 10^{-14} \mathrm{~m}^{2}$. This value correlates well with the bulk value of gold, $2.21 \times 10^{-8} \Omega \cdot \mathrm{m}$ (29) and the slightly lower resistivity measurement may be attributed to grain boundary scattering within the polycrystalline nanowires (30). Figure $2 \mathrm{c}$ shows a high resolution scanning electron micrograph of a portion of a nanowire with grain boundaries on the order of $32 \pm$ $2 \mathrm{~nm}$.

$\mathrm{CVs}$ in $0.1 \mathrm{M} \mathrm{H}_{2} \mathrm{SO}_{4}$ were employed as an electrode cleaning method because these nanowires cannot be polished like with macro-electrodes. A typical $\mathrm{H}_{2} \mathrm{SO}_{4}$ cyclic voltammogram $(\mathrm{CV})$ recorded using a typical gold nanowire electrode is shown in Figure 2b. The characteristic gold oxide formation peak (ca. $1.13 \mathrm{~V}$ ) and oxide reduction peaks (ca. $0.78 \mathrm{~V}$ ) are in good agreement with data in the literature (31). The charge for gold oxide monolayer reduction may be converted to the real surface area of $2.92 \times 10^{-7} \mathrm{~cm}^{2}$ using the conversion factor $386 \mu \mathrm{C} \mathrm{cm}^{-2}$ (32). In this manner, it was estimated that nanowires had an average roughness factor of $\sim 6$, i.e., the electrochemical active area was approximately 6 times higher than the geometric surface area; see Figure 2c. It would therefore be expected that this increased electrochemically active area would lead to a higher measurable electrochemical current obtained from individual nanowires.
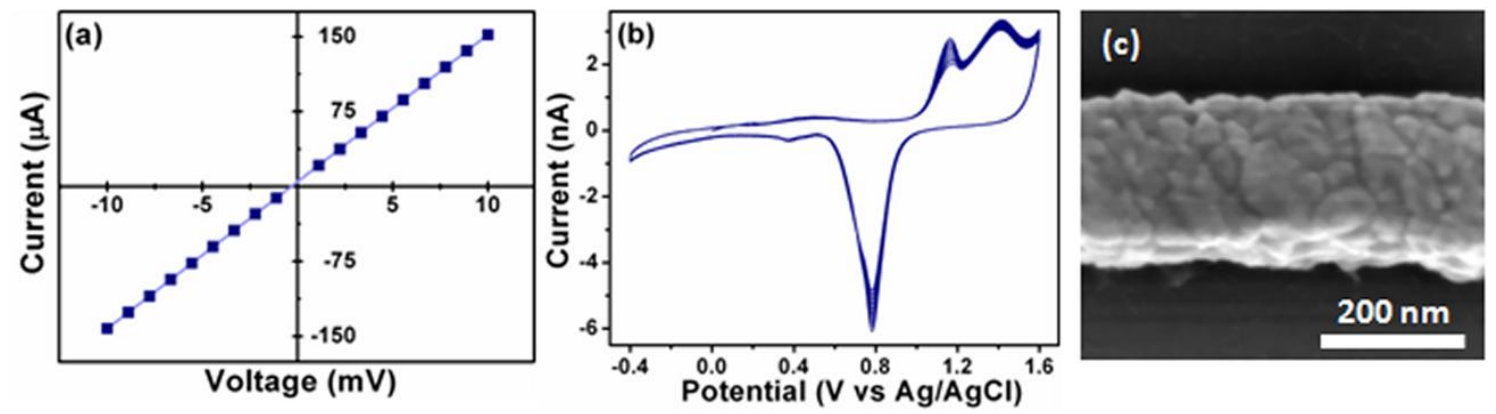

Figure 2. (a). Typical $I-V$ characteristic of a packaged nanowire exhibiting low resistance, $\sim 80 \Omega$. (b) $\mathrm{CV}$ of a typical gold nanowire in $0.1 \mathrm{M} \mathrm{H}_{2} \mathrm{SO}_{4}$, from -0.4 to +1.6 $\mathrm{V}$ at $100 \mathrm{mV} \mathrm{s}^{-1}$. (c) Scanning electron micrograph of a skived nanowire with high roughness.

This expectation was borne out during experiments using $1 \mathrm{mM}$ ferrocene monocarboxylic acid (FcCOOH) in $10 \mathrm{mM}$ PBS, pH 7.4 and $25^{\circ} \mathrm{C}$, see Figure 3a. To undertake experiments, a potentiostat (CHI 660) with a faraday cage ( $\mathrm{CH}$ Instruments, Texas) were employed to apply a potential scan range of $0.1-0.6 \mathrm{~V}$ to a nanowire working electrode with respect to $\mathrm{Ag} / \mathrm{AgCl}$ reference and with a $\mathrm{Pt}$ wire counter 
electrode. At high potential an anodic current corresponding to the oxidation of $\mathrm{FcCOOH}$ was observed. A sloped plateau region was not observed and the voltammetric waves appeared stretched. This may be attributed to heterogeneous electrode kinetics which control the rate of electrode reactions. Due to the small critical dimension of the $\mathrm{NW}$, mass transport is highly effective, and the rate of mass transport may become comparable to or larger than the rate of electron transfer. The magnitude of the diffusioncontrolled current was typically on the order of $1.5 \mathrm{nA}$. Control CV experiments were undertaken using control devices (without nanowires) in the presence of FcCOOH in PBS electrolyte solution. No electrochemical waves were observed during these control experiments indicating that the interconnection tracks were sufficiently insulated from the electrolytic solution. The magnitude of the steady state current was typically on the order of $1.5 \mathrm{nA}$ and was independent of scan rates up to $2 \mathrm{~V} / \mathrm{s}$; see Figure $3 \mathrm{~b}$.
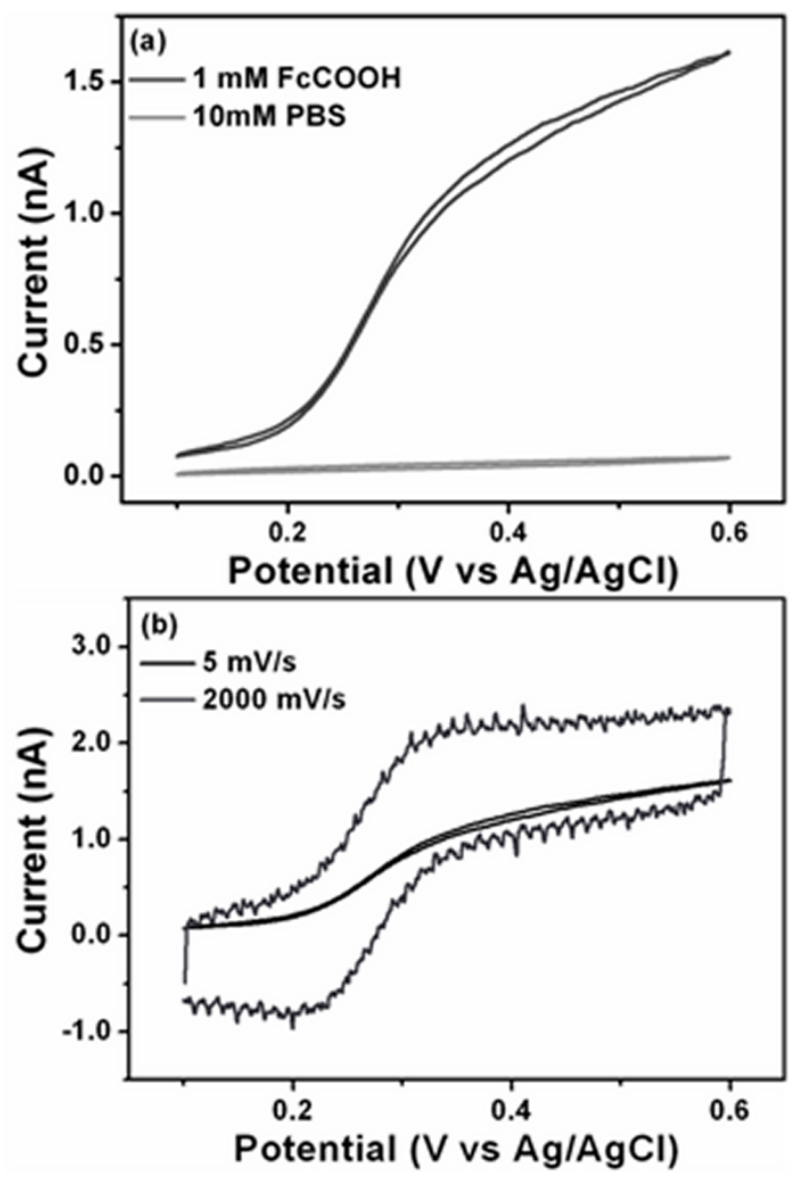

Figure 3. a) Cyclic Voltammograms obtained for a nanowire electrode in the presence (dark line) and absence (light line) of $1 \mathrm{mM} \mathrm{FcCOOH}$ in PBS, from $0.1-+0.6 \mathrm{~V}$, at $5 \mathrm{mV}$ $\mathrm{s}^{-1}$. (b) Cyclic Voltammograms exhibited steady-state voltammetric responses independent of scan rate from 5 to $2000 \mathrm{mVs}^{-1}$.

\section{Electrochemical Analysis:}

The enhanced mass transport rate at a nanoelectrode allows the kinetics of electrode reactions to be accessed through voltammetry. The classic Butler-Volmer model of electrode kinetics (33) was fitted to the CV data, see Figure 4. The classic Butler-Volmer current model for a one-step, one-electron oxidation reaction the oxidative current $i_{B V}$ may be defined as: 


$$
i_{B V}=\frac{i_{\mathrm{d}}}{1+\mathrm{e}^{-F\left(E-E^{0}\right) / R T}+K_{0}^{-1} \mathrm{e}^{-F(1-\alpha)\left(E-E^{0}\right) / R T}}
$$

where $i_{\mathrm{d}}$ is the diffusion-limited current, $F$ is the Faraday constant, $R$ is the molar gas constant, $T$ is temperature $(\mathrm{K}), E$ is the applied potential, $E^{0}$ is the formal potential of the redox-couple, $\alpha$ is the transfer coefficient, and $K_{0}=F A C k^{0} / i_{\mathrm{d}}$, where $A$ is the electrode surface area, $C$ is the bulk concentration of the electroactive species, and $k^{0}$ is the standard heterogeneous rate constant. Figure 4 shows a fit of Eq. 1 to the experimental data presented in Figure $3 a$ using $k^{0}, \alpha$, and $i_{d}$ as fitting parameters. Non-linear least square fitting of experimental voltammograms by Equation (1) was performed by the Levenberg-Marquardt algorithm (34). The fitting range was restricted to below a potentials below $0.4 \mathrm{~V}$, so that only the features of the voltammogram containing information about the electrode kinetics (i.e. the raising part) was included and the fit was not influenced by experimental artifacts (i.e. the experimental limiting current). The values for $E^{0^{\prime}}, C$ and $A$ were fixed; $E^{0^{\prime}}=0.306 \mathrm{mV}, C=1 \mathrm{mM}$ and $\mathrm{A}$ was calculated using the geometric area of the nanowire $=2.99 \times 10^{-11} \mathrm{~cm}^{2}$.

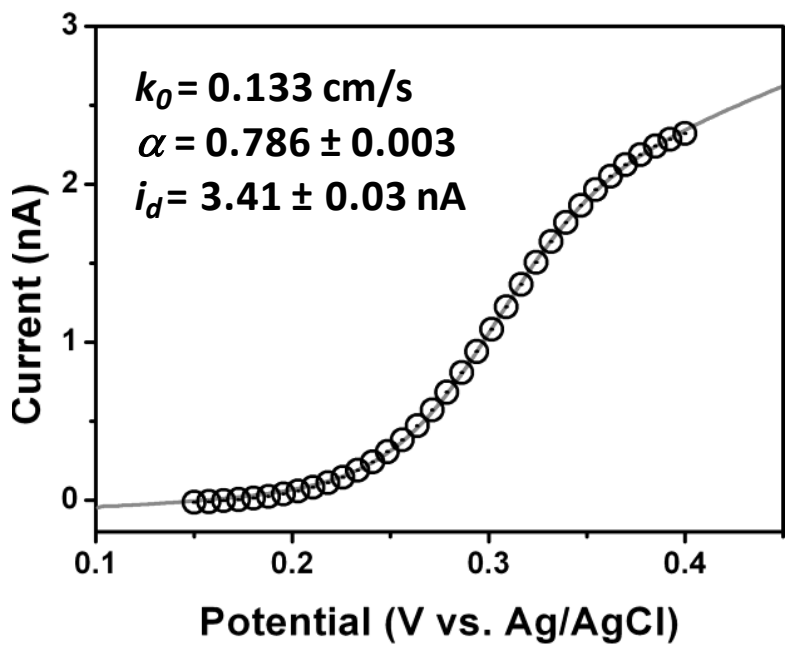

Figure 4. Fit of the Butler-Volmer model fit to $\mathrm{CV}$ data obtained using a nanoskived nanowire electrode.

\section{Electrochemical Sensing:}

To explore utility of nanowires for use in as electrochemical-based sensing applications, glucose was selected as a key target analyte. Glucose detection is of critical importance in a variety of application areas including: food industry for quality control purposes, in fermentation, and as a clinical indicator for diabetes. Direct observation of well defined electrochemical behavior from immobilized glucose oxidase (GOx) is difficult since the flavin adenine dinucleotide (FAD) redox center group is embedded deep within the protein structure (35). Therefore the electrons produced in the enzymecatalyzed reaction cannot always be easily and rapidly transferred to the electrode surface. One approach to surmount is to employ artificial redox mediators, small molecules, capable of undergoing rapid and reversible redox reactions, as shuttles to mediate the electronic transfer between the redox center of the enzyme and the electrode surface (35-37). We employed $0.1 \mathrm{mM} \mathrm{FcCOOH}$ to mediate the oxidation of glucose, in 
the presence of $1 \mathrm{mg} / \mathrm{ml}$ soluble GOx, to a nanowire electrode; see Figure 5a. Experiments were performed using different concentrations of glucose in the physiologically important concentration range of $1-15 \mathrm{mM}$. CVs corresponding to the bio-electrocatalytic oxidation of glucose presented in Figure 5b show a well defined increase in catalytic current with increasing glucose concentration. Although work is ongoing to optimize sensor design and assay parameters in terms of reproducibility, repeatability accuracy etc., these results demonstrate the clear potential of nanowire electrodes for use in future up-stream electrochemical-based diagnostic devices.
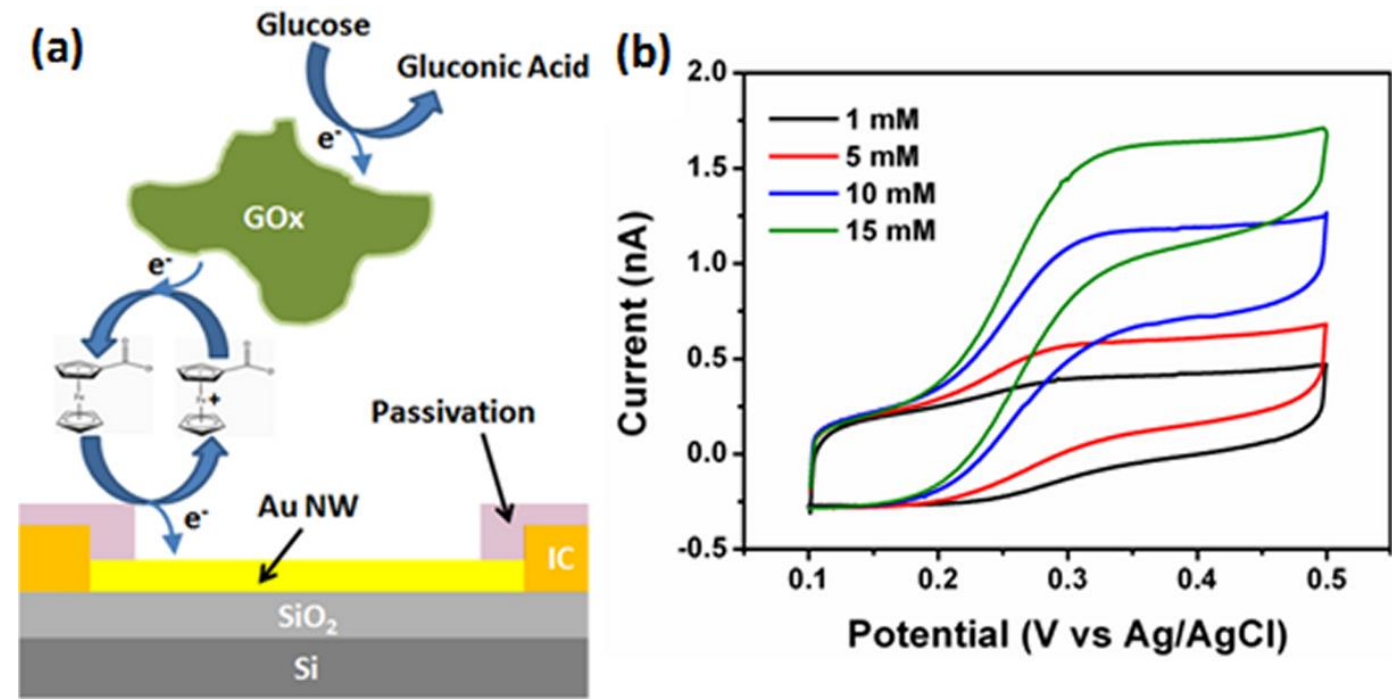

Figure 5. Schematic of the reversible electrochemical oxidation of FcCOOH coupled glucose oxidation biocatalyzed by GOx in PBS from $0.1-+0.5 \mathrm{~V}$, at $5 \mathrm{mVs}^{-1}$.

\section{Conclusion:}

Individual gold nanowire devices for use as nanoelectrodes in electrochemical studies were fabricated using a low cost robust fabrication approach. Nanowires were characterized by a combination of direct electrical current-voltage (I-V) measurements and cyclic voltammetry $(\mathrm{CV})$ to determine their functionality. We observed low electrical resistances to contacted nanowires and diffusion-controlled electrochemical voltammograms that may be described by classical Butler-Volmer kinetics. We explored the potential of these nanowire electrodes for use as future electrochemical sensor devices by employing FcCOOH to mediate the oxidation of glucose in the presence of soluble GOx at a nanowire electrode.

\section{Acknowledgments:}

This work was supported by the European Commission under the FP6 NMP project "Hydromel" (NMP2-CT-2006-026622), by Science Foundation Ireland under the Research Frontiers Programme (SFI/09/RFP/CAP2455) and the Irish HEA PRTLI program (Cycle 3 "Nanoscience" and Cycle 4 "Inspire").

\section{References:}

1. Frost \& Sullavin, (2007).

2. F. Patolsky, G. F. Zheng and C. M. Lieber, Nature Protocols, 1, 1711 (2006). 
3. M. Curreli, C. Li, Y. H. Sun, B. Lei, M. A. Gundersen, M. E. Thompson and C. W. Zhou, Journal of the American Chemical Society, 127, 6922 (2005).

4. K. Maehashi, T. Katsura, K. Kerman, Y. Takamura, K. Matsumoto and E. Tamiya, Analytical Chemistry, 79, 782 (2007).

5. E. Stern, J. F. Klemic, D. A. Routenberg, P. N. Wyrembak, D. B. Turner-Evans, A. D. Hamilton, D. A. LaVan, T. M. Fahmy and M. A. Reed, Nature, 445, 519 (2007).

6. D. W. M. Arrigan, Analyst, 129, 1157 (2004).

7. R. M. Penner, M. J. Heben, T. L. Longin and N. S. Lewis, Science, 250, 1118 (1990).

8. V. P. Menon and C. R. Martin, Analytical Chemistry, 67, 1920 (1995).

9. W. L. Cheng, S. J. Dong and E. K. Wang, Analytical Chemistry, 74, 3599 (2002).

10. C. W. Welch and R. G. Compton, Analytical and Bioanalytical Chemistry, 384, $601(2006)$.

11. S. N. Aravamudhan, S.R., Bhansali, S., Sensors and Actuators B, 127, 29 (2007).

12. E. C. N. Walter, K.; Zach, M.P.; Penner, R.M.; Favier, F., Microelectronic engineering, 61-62, 55 (2002).

13. R. Baron, F. W. Campbell, I. Streeter, L. Xiao and R. G. Compton, International Journal of Electrochemical Science, 3, 556 (2008).

14. R. Baron, B. Sljukic, C. Salter, A. Crossley and R. G. Compton, Electroanalysis, 19, 1062 (2007).

15. S. E. Creager and P. T. Radford, Journal of Electroanalytical Chemistry, 500, 21 (2001).

16. W. S. Baker and R. M. Crooks, Journal of Physical Chemistry B, 102, 10041 (1998).

17. E. Jeoung, T. H. Galow, J. Schotter, M. Bal, A. Ursache, M. T. Tuominen, C. M. Stafford, T. P. Russell and V. M. Rotello, Langmuir, 17, 6396 (2001).

18. Y. H. Lanyon, G. De Marzi, Y. E. Watson, A. J. Quinn, J. P. Gleeson, G. Redmond and D. W. M. Arrigan, Analytical Chemistry, 79, 3048 (2007).

19. N. Godino, X. Borrise, F. X. Munoz, F. J. del Campo and R. G. Compton, Journal of Physical Chemistry C, 113, 11119 (2009).

20. M. P. Nagale and I. Fritsch, Analytical Chemistry, 70, 2902 (1998).

21. S. L. Chen and A. Kucernak, Journal of Physical Chemistry B, 106, 9396 (2002).

22. S. L. Chen and A. Kucernak, Electrochemistry Communications, 4, 80 (2002).

23. J. L. Conyers and H. S. White, Analytical Chemistry, 72, 4441 (2000).

24. M. V. Mirkin, F. R. F. Fan and A. J. Bard, Journal of Electroanalytical Chemistry, 328, 47 (1992).

25. D. K. Y. Wong and L. Y. F. Xu, Analytical Chemistry, 67, 4086 (1995).

26. Y. H. Shao, M. V. Mirkin, G. Fish, S. Kokotov, D. Palanker and A. Lewis, Analytical Chemistry, 69, 1627 (1997).

27. B. B. Katemann and T. Schuhmann, Electroanalysis, 14, 22 (2002).

28. Q. B. Xu, R. Perez-Castillejos, Z. F. Li and G. M. Whitesides, Nano Lett., 6, 2163 (2006).

29. C. M. Lilley and Q. J. Huang, Applied Physics Letters, 89 (2006).

30. G. D. Marzi, D. Iacopino, A. J. Quinn and G. Redmond, Journal of Applied Physics, 96, 3458 (2004).

31. A. Berduque, Y. H. Lanyon, V. Beni, G. Herzog, Y. E. Watson, K. Rodgers, F. Stam, J. Alderman and D. W. M. Arrigan, Talanta, 71, 1022 (2007).

32. $\quad$ L. D. Burke and P. F. Nugent, Gold Bull., 31, 39 (1998). 
33. I. Heller, J. Kong, H. A. Heering, K. A. Williams, S. G. Lemay and C. Dekker, Nano Lett., 5, 137 (2005).

34. B. P. F. W. H. Press, S. A. Teukolsky, W. T. Vetterling, Cambridge University Press (1992).

35. N. J. Ronkainen, H. B. Halsall and W. R. Heineman, Chemical Society Reviews, 39, 1747 (2010).

36. J. Wang, Chemical Reviews, 108, 814 (2008).

37. J. Jimenez, R. Sheparovych, M. Pita, A. Narvaez Garcia, E. Dominguez, S. Minko and E. Katz, The Journal of Physical Chemistry C, 112, 7337 (2008). 